Revista Brasil. Bot., V.28, n.2, p.409-418, abr.-jun. 2005

\title{
Sistema de reprodução em Caesalpinia echinata Lam. implantada em arboreto experimental
}

\author{
JOÃO DEL GIUDICE NETO ${ }^{1,4}$, ALEXANDRE MAGNO SEBBENN² e PAULO Y. KAGEYAMA ${ }^{3}$
}

(recebido: 15 de abril de 2004; aceito: 11 de março de 2005)

\begin{abstract}
Mating system in Caesalpinia echinata Lam. implanted in experimental arboretum). The mating system of Caesalpinia echinata Lam. implanted in arboretum was studied by allozymes analysis of progeny arrays using the mixedmating model and correlated-mating model. Deviations from mixed-mating model were observed from heterogeneity of pollen pools allele frequencies that fertilized different trees. The multilocus outcrossing rate was high $\left(\hat{\mathrm{t}}_{\mathrm{m}}=0.969\right)$, indicating that the species is predominantly allogamous. The high variation observed in the individual outcrossing rate $(\hat{\mathrm{t}}$ ranged from 0.77 to 1.00) shows that the species is not auto-incompatible. It was detected significant positive difference between multilocus and single locus outcrossing rate suggesting that biparental inbreeding has occurred $\left(\hat{t}_{m}-\hat{t}_{s}=0.078\right)$. High value of paternity correlation was detected $\left(\hat{r}_{\mathrm{p}}=0.822\right)$, demonstrating that families are composed by full sibs, mainly. The coefficient of coancestry within families $\left(\hat{\theta}_{x y}=0.269\right)$ was higher than expected in half-sibs families $(0.125)$. The results are discussed in the viewpoint of the sampling to genetic conservation and environmental recovering.
\end{abstract}

Key words - biparental inbreeding, brazilwood, coancestry, effective variance size, outcrossing rate

RESUMO - (Sistema de reprodução em Caesalpinia echinata Lam. implantada em arboreto experimental). O sistema de reprodução de Caesalpinia echinata Lam. foi estudado por isoenzimas em arboreto, usando os modelos de cruzamentos mistos e cruzamentos correlacionados. Desvios do modelo misto de reprodução foram evidenciados pela heterogeneidade nas frequiências alélicas do pólen que fecundou as diferentes árvores. A taxa de cruzamento multiloco foi alta $\left(\hat{\mathrm{t}}_{\mathrm{m}}=0,969\right)$, indicando que a espécie é predominantemente alógama. A alta variação na taxa de cruzamento individual ( $\hat{\mathrm{t}}$ variando de 0,77 a 1,00$)$ indica que a espécie não é auto-incompatível. Diferença positiva e significativamente diferente de zero foi detectada entre as taxas de cruzamento multiloco e uniloco, sugerindo que ocorreram cruzamentos endogâmicos na população $\left(\hat{t}_{m}-\hat{t}_{s}=0,078\right)$. A correlação de paternidade foi alta $\left(\hat{\mathrm{r}}_{\mathrm{p}}=0,822\right)$, demonstrando que as progênies são constituídas principalmente por irmãos-completos. $\mathrm{O}$ coeficiente de coancestria nas progênies foi alto $\left(\hat{\theta}_{x y}=0,269\right)$ e superior ao esperado em progênies de meios-irmãos $(0,125)$. Os resultados são discutidos em termos de amostragens para a conservação genética e coleta de sementes para a recuperação ambiental.

Palavras-chave - coancestria, cruzamentos endogâmicos, pau-brasil, tamanho efetivo de variância, taxa de cruzamento

\section{Introdução}

Caesalpinia echinata Lam. (Leguminosae Caesalpinioideae), pau-brasil, foi a primeira espécie arbórea brasileira a ser explorada comercialmente em larga escala. Contudo, hoje, ela está na lista das espécies arbóreas brasileiras ameaçadas de extinção (Brasil 1992). A exploração intensiva desde o descobrimento do Brasil, quando era encontrada em abundância,

1. Instituto de Botânica, Caixa Postal 4005, 01061-970 São Paulo, SP, Brasil.

2. Instituto Florestal de São Paulo, Caixa Postal 1322, 01059-970 São Paulo, SP, Brasil.

3. Escola Superior de Agricultura Luiz de Queiroz, Departamento de Ciências Florestais, Av. Pádua Dias 11, 13418-900 Piracicaba, SP, Brasil.

4. Autor para correspondência: jdgiudic@ig.com.br levou-a quase ao desaparecimento em conseqüência da devastação da Mata Atlântica. Atualmente, na sua área de ocorrência natural, que vai do Estado do Rio Grande do Norte $\left(05^{\circ} 45^{\prime} \mathrm{S}\right)$ ao Estado do Rio de Janeiro $\left(23^{\circ} \mathrm{S}\right)$, ainda persistem algumas pequenas populações fragmentadas. Assim, necessita-se estabelecer um trabalho amplo de conservação para a espécie, principalmente in situ (Carvalho 1994).

A conservação in situ é considerada ideal para as espécies arbóreas, contudo, há algumas situações em que a conservação ex situ é essencial e complementar àquela in situ. A situação mais flagrante dessa complementação é a que se refere às espécies raras em risco de extinção (Kageyama 1987), sendo a C. echinata um exemplo clássico desse tipo de espécie.

A conservação genética de uma espécie requer o conhecimento de seu sistema de reprodução, variabilidade e estrutura genética. O sistema de reprodução é um importante determinante da estrutura genética e herança evolutiva de populações por 
estabelecer os padrões de como os gametas são unidos para formar a próxima geração (Allard 1971). Ele pode ser eficientemente estudado por meio de marcadores bioquímicos, como isoenzimas. Estudos baseados em isoenzimas têm detectado amplas variações na taxa de cruzamento entre espécies arbóreas tropicais (Murawski \& Hamrick 1991), populações (Lee 2000), indivíduos dentro de populações (Burgess et al. 1996, Sebbenn et al. 2000, Souza et al. 2003) e diferentes eventos reprodutivos (Murawski et al. 1990). Estas variações podem ser o resultado de diferentes fatores ecológicos, tais como tamanho e densidade da população, densidade de florescimento das árvores, fenologia de florescimento, comportamento dos polinizadores, vetor de polinização (Murawski \& Hamrick 1991, Murawski 1995) e devido a fatores genéticos, como a variação individual na auto-incompatibilidade.

Não existem relatos na literatura de estimativas de taxa de cruzamento em populações de $C$. echinata. Assim, o objetivo deste trabalho foi analisar os padrões de reprodução em uma população ex situ de C. echinata, usando a eletroforese de isoenzimas e os modelos de reprodução mista e de cruzamentos correlacionados.

\section{Material e métodos}

Área de estudo e amostragem - $\mathrm{O}$ estudo foi realizado no arboreto experimental de pau-brasil $\left(22^{\circ} 15^{\prime} 02,4^{\prime \prime} \mathrm{S}\right.$ e $47^{\circ} 09^{\prime} 28,9^{\prime \prime} \mathrm{W}$; altitude média $650 \mathrm{~m}$ ) implantado na Reserva Biológica e Estação Experimental do Instituto de Botânica da Secretaria do Meio Ambiente do Estado de São Paulo ( $22^{\circ} 14,8^{\prime}$ $15,6^{\prime} \mathrm{S}$ e $47^{\circ} 8,8^{\prime}-11,6^{\prime} \mathrm{W}$, altitude média de $640 \mathrm{~m}$ ), no município de Mogi-Guaçu. A área total do arboreto é de $3.000 \mathrm{~m}^{2}$, aproximadamente, tendo sido implantado em dezembro de 1980, a partir de mudas originadas de sementes provenientes da Estação Ecológica de Tapacurá, Estado de Pernambuco, dentro da zona de ocorrência natural da espécie (Aguiar 1992). Não existem informações sobre o número de árvores matrizes que deu origem às sementes utilizadas no plantio do arboreto. Inicialmente, sua implantação visava testar o efeito de diferentes espaçamentos sobre as características dendrométricas da espécie. Mas, devido ao quadro alarmante de erosão genética em que a espécie se encontra, esse arboreto passou a ser uma importante fonte potencial de material para a recombinação e uso da sua variabilidade genética. Atualmente, o arboreto tem 262 árvores com altura média de doze metros e diâmetro à altura do peito (DAP) em torno de $17 \mathrm{~cm}$. O espaçamento médio atual entre as árvores é $3,5 \mathrm{~m} \times 3,5 \mathrm{~m}$.

Para a análise genética de C. echinata, em 1999 foram coletadas sementes de polinização aberta em 27 árvores do arboreto, dentre as que produziram frutos no período de amostragem. As sementes foram germinadas em substrato contendo uma mistura igual de terra vegetal, vermiculita e acícula de Pinus sp. peneirada. Tubetes com capacidade de $100 \mathrm{~cm}^{3}$ foram utilizados como recipientes. As mudas foram mantidas nesse tipo de recipiente durante toda a fase de estudo, recebendo, entretanto, adubação mineral de manutenção, a cada sete dias aproximadamente. Alternava-se a aplicação de nitrogênio ( $2 \mathrm{mg}$ de N.muda ${ }^{-1}$ ) e potássio ( $3 \mathrm{mg}$ de $\mathrm{K}_{2} \mathrm{O}$.muda ${ }^{-1}$ ).

Eletroforese de isoenzimas - Em janeiro de 2002 foram coletadas folhas de 31 árvores adultas e de 10 plântulas (progênies) de 27 dessas árvores. Nos adultos procurou-se coletar folhas jovens. Nas plântulas coletaram-se folhas do terceiro par ou superior. O período de coletas foi na parte da manhã, em dias chuvosos ou na ausência de sol, proporcionados pela época do ano. As amostras, depois de identificadas e acondicionadas em sacos de papel Kraft, foram armazenadas em geladeira a $5{ }^{\circ} \mathrm{C}$ por aproximadamente 24 horas, sendo então transportadas ao laboratório, onde foram submetidas às técnicas de eletroforese de isoenzimas, segundo as recomendações descritas em Soltis \& Soltis (1989), Kephart (1990) e Alfenas (1998). As corridas de eletroforese foram conduzidas em meio suporte de gel de amido a $13 \%$ (34,29 $\mathrm{g}$ de penetrose 30 e $18,29 \mathrm{~g}$ de amido de milho para $400 \mathrm{ml}$ de tampão) mais sacarose $(12,00 \mathrm{~g})$. A solução de extração utilizada foi a ${ }^{\circ} 1$ de Alfenas (1998) e o sistema de cuba e gel foi o Tris-Citrato (ácido cítrico $0,086 \mathrm{M}$ mais Tris $0,223 \mathrm{M}) \mathrm{pH} 7,5$. As sessões duravam em média quatro horas com uma intensidade elétrica constante de 80 miliamperes. Dezoito locos puderam ser interpretados em onze sistemas enzimáticos previamente selecionados (LAP - E.C. 3.4.11.1; EST-E.C. 3.1.1.1; ACP-E.C. 3.1.3.2; MDH -E.C. 1.1.1.37, três locos; 6PGDH - E.C. 1.1.1.44, dois locos; DIA - E.C. 1.8.1.4; PGI-E.C. 5.3.1.9, dois locos; G6PDH-E.C. 1.1.1.49; GOT - E.C. 2.6.1.1.; PRX - E.C. 1.11.1.7, quatros locos e SKDH - E.C. 1.1.1.25). Prévio estudo da herança e equilíbrio de ligação (Giudice-Neto et al. 2004) indicou que os locos Pgi-2, Prx-2 e Prx-4 não segregaram de forma mendeliana e, por isso, foram excluídos da análise.

Análise estatística - O sistema de reprodução foi analisado com base nos modelos de reprodução mista (Ritland \& Jain 1981) e cruzamentos correlacionados (Ritland 1989), usando o programa "Multilocos MLTR" (Ritland 1994). Os parâmetros estimados foram: i) a taxa populacional de cruzamento multiloco $\left(\hat{\mathrm{t}}_{\mathrm{m}}\right)$, pelo método de máxima verossimilhança (Expectation-Maximization); ii) a taxa populacional de cruzamento uniloco $\left(\hat{\mathrm{t}}_{\mathrm{s}}\right)$; iii) a taxa de cruzamento entre aparentados $\left(\hat{\mathrm{t}}_{\mathrm{m}}-\hat{\mathrm{t}}_{\mathrm{s}}\right)$; iv) a taxa individual de cruzamento multiloco $(\hat{t}) ; v)$ as freqüências alélicas dos óvulos e do pólen (o e p); vi) a correlação de autofecundação $\left(\hat{\mathrm{r}}_{\mathrm{s}}\right)$; vii) a correlação de paternidade $\left(\hat{r}_{\mathrm{p}}\right)$. As pressuposições do modelo misto são dadas em Ritland \& Jain (1981). O erro padrão das estimativas dos parâmetros foi obtido por meio de 500 reamostragens "bootstraps". A avaliação de cruzamentos 
aleatórios foi realizada pelo teste de homogeneidade entre as freqüências alélicas dos óvulos e do pólen e, entre as freqüências alélicas do pólen que fecundou cada árvore matriz, calculando-se o estimador $\hat{\mathrm{F}}_{\mathrm{ST}}$ (Nei 1977). A significância estatística de $\hat{\mathrm{F}}_{\mathrm{ST}}$ foi obtida para cada loco pelo teste de qui-quadrado, $\mathrm{F}^{2}=2 \mathrm{n}_{\mathrm{ST}}(\mathrm{k}-1)$, com $(\mathrm{k}-1)(\mathrm{s}-1)$ graus de liberdade, proposto por Workman \& Niswander (1970), em que: $\mathrm{n}=$ número de indivíduos nos grupos, $\mathrm{k}=$ número de alelos e $\mathrm{s}=$ número de grupos. Os índices de fixação para as árvores adultas $(\hat{\mathrm{F}})$ e progênies $\left(\hat{\mathrm{F}}_{\mathrm{p}}\right)$ e seus respectivos intervalos de confiança a $95 \%$ de probabilidade foram obtidos por 10.000 reamostragens "bootstraps". Os índices de fixação e as estimativas foram obtidos usando o programa GDA (Lewis \& Zaykin 2002).

O coeficiente de coancestria $\left(\hat{\theta}_{x y}\right)$ entre plantas dentro de progênies foi estimado do coeficiente de correlação de parentesco $\left(\hat{\mathrm{r}}_{\mathrm{xy}}\right)$ entre plantas dentro de progênies, derivado por Ritland (1989) de parâmetros do sistema de reprodução, segundo o qual $\hat{\mathrm{r}}_{\mathrm{xy}}=0,25\left(1+\hat{\mathrm{F}}_{\mathrm{a}}\right)\left[4 \hat{\mathrm{s}}+\left(\hat{\mathrm{t}}^{2}+\hat{\mathrm{t}} \hat{\mathrm{s}} \hat{\mathrm{s}}_{\mathrm{s}}\right)\left(1+\hat{\mathrm{r}}_{\mathrm{p}}\right)\right]$ em que, $\hat{\mathrm{F}}_{\mathrm{a}}$ é o coeficiente de endogamia na geração parental e $\hat{s}$ é a taxa de autofecundação ( $\left.\begin{array}{ll}\hat{\mathrm{s}} 1 \square \hat{\mathrm{t}}_{\mathrm{m}}\end{array}\right)$. Como em espécies diplóides, na ausência de endogamia, o coeficiente de parentesco $\left(\hat{r}_{x y}\right)$ é o dobro do coeficiente de coancestria $\left(\hat{\theta}_{x y}\right)$, tem-se que, $\hat{\theta}_{x y}$ $=\hat{\mathrm{r}}_{\mathrm{xy}} / 2$ e pode-se, portanto, obter o coeficiente de coancestria do coeficiente de parentesco. $\mathrm{O}$ tamanho efetivo de variância ( $\hat{\mathrm{N}}_{\mathrm{e}(\mathrm{v})}$ ) de uma simples progênie foi estimado com base na variância amostral de um alelo, segundo derivações de Cockerham (1969),

$$
\hat{\mathrm{N}}_{\mathrm{e}(\mathrm{v})}=\frac{0,5}{\hat{\theta}_{\mathrm{xy}}\left(\frac{\mathrm{n}-1}{\mathrm{n}}\right)+\frac{1+\hat{\mathrm{F}}_{\mathrm{p}}}{2 \mathrm{n}}}
$$

em que n é o número total de progênies na população, assumido como infinito, $\hat{\mathrm{F}}_{\mathrm{p}}$ é o coeficiente de endogamia do conjunto de progênies, e, utilizando uma população idealizada como referência, como descrito em Sebbenn (2002).

\section{Resultados e Discussão}

Frequiências alélicas - As freqüências alélicas do pólen e dos óvulos foram estatisticamente diferentes em quatro dos quinze locos avaliados (tabela 1). $\mathrm{O}$ teste de quiquadrado detectou que as frequiências alélicas do pólen e dos óvulos que contribuíram para a formação das progênies amostradas de $C$. echinata diferiram estatisticamente nos locos Dia-1, Mdh-1, Prx-3 e Skdh-1 $(\mathrm{p}<0,01)$. O teste de qui-quadrado para as frequiências alélicas do conjunto de pólen que fecundou cada árvore materna individualmente detectou diferenças altamente significativas $(\mathrm{p}<0,01)$ para nove locos (Acp-1, Dia-1, Est-3, Mdh-1, Mdh-3, Prx-1, Prx-3, Skdh-1 e 6pgdh-2) e p $<0,05$ para o Lap-1 (tabela 2), indicando que o pólen cruzado não foi homogêneo entre diferentes árvores maternas. Estes resultados sugerem que os cruzamentos no arboreto não foram aleatórios. O teste de quiquadrado para as diferenças entre as frequiências alélicas do pólen cruzado e as árvores adultas amostradas no arboreto detectou diferenças significativas nos locos Dia-1, Mdh-1, Prx-1, Prx-3, Skdh-1 e 6pgdh-1 (p <0,01) e nos locos Acp-1 e G6pdh-1 (p < 0,05) (tabela 2), sugerindo que as freqüências alélicas da população paterna que polinizou as progênies amostradas não representam as frequiências alélicas das árvores adultas ou, em outras palavras, apenas parte da população participou, efetivamente, do evento reprodutivo, sofrendo com isso dos efeitos de deriva genética.

Diferenças nas frequiências alélicas entre pólen e óvulos podem ser causadas por diversos fatores como diferenças nas funções masculina e feminina das árvores, migração de pólen de fora da população, seleção entre o período de polinização e amostragem das progênies ou devido à reprodução não aleatória dos genótipos da população como, por exemplo, cruzamentos endogâmicos, cruzamentos biparentais e autofecundações, ou ainda, pelo baixo tamanho amostral no número de plantas maternas ou desigual número de plantas acessadas por progênies. No presente estudo, exclui-se a possibilidade de migração de pólen de fora do plantio, haja vista que não existem outras populações da espécie no local ou próximas ao arboreto. Entretanto, dado que apenas quatro locos, entre quinze, apresentaram desvios significativos entre óvulos e pólen, pode-se concluir pela não diferença entres as frequiências destes grupos.

As divergências genéticas, estimadas pelo $\hat{\mathrm{F}}_{\mathrm{ST}}$, entre as freqüências alélicas do pólen que fecundou as árvores maternas indicam que as árvores não receberam pólen aleatório da população (arboreto). A heterogeneidade nas frequiências do pólen pode ser causada por variações na taxa de autofecundação entre árvores, estruturação na população que pode gerar cruzamentos entre parentes e criar heterogeneidade genética espacial no conjunto de pólen liberado ou, devido à ineficiência do polinizador em homogeneizar o pólen liberado, favorecendo cruzamentos sistemáticos na população (cruzamentos biparentais). Outra causa pode ser o assincronismo no florescimento, de forma que as árvores maternas só receberam pólen de árvores que o estavam liberando ao mesmo tempo.

Taxas de cruzamento multiloco e uniloco - A estimativa da taxa de cruzamento multiloco populacional $\left(\hat{\mathrm{t}}_{\mathrm{m}}\right)$ foi de 0,969 (tabela 3), demonstrando que a espécie C. echinata encontra-se na classe das 
Tabela 1. Estimativa da divergência genética $\left(\hat{\mathrm{F}}_{\mathrm{ST}}\right)$ entre as freqüências alélicas do pólen e dos óvulos, tamanho amostral (n) e teste de qui-quadrado $\left(\mathrm{F}^{2}\right)$ em Caesalpinia echinata Lam. implantada em arboreto experimental (GL = grau de liberdade; $* * \mathrm{p}<0,01 ; * \mathrm{p}<0,05)$.

Table 1. Estimation of genetic divergence $\left(\hat{\mathrm{F}}_{\mathrm{ST}}\right)$ between allelic frequencies of pollen and ovule pools, sample size $(\mathrm{n})$ and chisquare test $\left(\mathrm{F}^{2}\right)$ in Caesalpinia echinata $\mathrm{Lam}$. implanted in experimental arboretum $\left(\mathrm{GL}=\right.$ freedom degree; $\left.* * \mathrm{p}<0.01 ;{ }^{*} \mathrm{p}<0.05\right)$.

\begin{tabular}{|c|c|c|c|c|c|c|c|}
\hline Loco & Alelo & Pólen & Óvulo & $\hat{\mathrm{F}}_{\mathrm{ST}}$ & $\mathrm{n}$ & $\mathrm{F}^{2}$ & GL \\
\hline \multirow[t]{2}{*}{ Acp-1 } & 1 & 0,228 & 0,204 & & & & \\
\hline & 2 & 0,772 & 0,796 & 0,001 & 218 & 0,37 & 1 \\
\hline \multirow[t]{3}{*}{ Dia-1 } & 1 & 0,005 & 0,019 & & & & \\
\hline & 2 & 0,326 & 0,648 & & & & \\
\hline & 3 & 0,669 & 0,333 & 0,106 & 211 & $89,39 * *$ & 2 \\
\hline \multirow{2}{*}{ Est-3 } & 1 & 0,513 & 0,556 & & & & \\
\hline & 2 & 0,487 & 0,444 & 0,002 & 208 & 0,77 & 1 \\
\hline \multirow[t]{2}{*}{ Got-1 } & 1 & 0,996 & 0,982 & & & & \\
\hline & 2 & 0,004 & 0,018 & 0,005 & 233 & 2,10 & 1 \\
\hline \multirow[t]{2}{*}{ G6pdh-1 } & 1 & 0,980 & 0,982 & & & & \\
\hline & 2 & 0,020 & 0,018 & 0,000 & 213 & 0,02 & 1 \\
\hline \multirow[t]{2}{*}{ Lap-1 } & 1 & 0,684 & 0,648 & & & & \\
\hline & 2 & 0,316 & 0,352 & 0,001 & 117 & 0,34 & 1 \\
\hline \multirow[t]{2}{*}{ Mdh-1 } & 1 & 0,562 & 0,074 & & & & \\
\hline & 2 & 0,438 & 0,926 & 0,275 & 233 & $127,92 * *$ & 1 \\
\hline \multirow[t]{2}{*}{ Mdh-2 } & 1 & 0,996 & 0,982 & & & & \\
\hline & 2 & 0,004 & 0,018 & 0,005 & 233 & 2,10 & 1 \\
\hline \multirow[t]{3}{*}{ Mdh-3 } & 1 & 0,069 & 0,037 & & & & \\
\hline & 2 & 0,365 & 0,352 & & & & \\
\hline & 3 & 0,566 & 0,611 & 0,002 & 227 & 1,40 & 2 \\
\hline \multirow[t]{2}{*}{ Pgi-1 } & 1 & 0,996 & 0,982 & & & & \\
\hline & 2 & 0,004 & 0,018 & 0,005 & 232 & 2,09 & 1 \\
\hline \multirow[t]{3}{*}{ Prx-1 } & 1 & 0,325 & 0,389 & & & & \\
\hline & 2 & 0,348 & 0,407 & & & & \\
\hline & 3 & 0,327 & 0,204 & 0,009 & 131 & 4,51 & 2 \\
\hline \multirow[t]{3}{*}{ Prx-3 } & 1 & 0,302 & 0,426 & & & & \\
\hline & 2 & 0,397 & 0,241 & & & & \\
\hline & 3 & 0,301 & 0,333 & 0,015 & 191 & $11,70^{* * *}$ & 2 \\
\hline \multirow[t]{2}{*}{ Skdh-1 } & 1 & 0,769 & 0,926 & & & & \\
\hline & 2 & 0,231 & 0,074 & 0,048 & 169 & $16,12^{* * *}$ & 1 \\
\hline \multirow[t]{3}{*}{ 6pgdh-1 } & 1 & 0,978 & 0,964 & & & & \\
\hline & 2 & 0,018 & 0,018 & & & & \\
\hline & 3 & 0,004 & 0,018 & 0,002 & 233 & 1,61 & 2 \\
\hline \multirow[t]{3}{*}{$6 p g d h-2$} & 1 & 0,086 & 0,056 & & & & \\
\hline & 2 & 0,507 & 0,481 & & & & \\
\hline & 3 & 0,407 & 0,463 & 0,002 & 230 & 1,93 & 2 \\
\hline
\end{tabular}

predominantemente de cruzamento. Pelo erro padrão da estimativa da taxa $\hat{\mathrm{t}}_{\mathrm{m}}$, verifica-se que esta é estatisticamente diferente da unidade $(1,0)$. A estimativa da taxa de cruzamento multiloco individual $(\hat{\mathrm{t}})$ por árvore materna (figura 1) foi, geralmente, alta, variando de 0,77 a 1,0, sendo que em 24 das 27 árvores (89\%) a taxa de cruzamento foi igual ou maior do que 0,85 . A taxa de cruzamento uniloco populacional $\left(\hat{\mathrm{t}}_{\mathrm{s}}\right)$ foi igualmente alta $(0,891)$ (tabela 3$)$, embora tenha sido significativamente diferente da taxa de cruzamento multiloco, a julgar pelo erro associado. A diferença entre a taxa de cruzamento multiloco e a uniloco $\left(\hat{t}_{\mathrm{m}}-\hat{\mathrm{t}}_{\mathrm{s}}\right)$ foi de 0,078 (tabela 3 ). Considerando o erro associado à estimativa de $\hat{t}_{m}-\hat{t}_{s}$ observa-se que ela foi significativamente diferente de zero, sugerindo a ocorrência de cruzamentos entre indivíduos aparentados e a presença de endogamia biparental na população descendente.

Não existem relatos de estimativas da taxa de 
Tabela 2. Estimativa da divergência genética $\left(\hat{\mathrm{F}}_{\mathrm{ST}}\right)$ entre as frequiências alélicas do pólen em diferentes árvores e entre o pólen e as árvores adultas amostradas em Caesalpinia echinata Lam. implantada em arboreto experimental (GL = grau de liberdade; $* * \mathrm{p}<0,01 ; * \mathrm{p}<0,05)$.

Table 2. Estimation of genetic divergence $\left(\hat{\mathrm{F}}_{\mathrm{ST}}\right)$ between allelic frequencies of pollen pools from different trees and between adult trees and pollen pool in Caesalpinia echinata $\mathrm{Lam}$. implanted in experimental arboretum $(\mathrm{GL}=$ freedom degree; $* * \mathrm{p}<0.01$; $* \mathrm{p}<0.05)$.

\begin{tabular}{|c|c|c|c|c|c|c|c|c|}
\hline \multirow[b]{2}{*}{ Loco } & \multicolumn{4}{|c|}{ Heterogeneidade do pólen } & \multicolumn{4}{|c|}{ Árvores adultas vs pólen } \\
\hline & $\hat{\mathrm{F}}_{\mathrm{ST}}$ & $\mathrm{n}$ & $\mathrm{F}^{2}$ & GL & $\hat{\mathrm{F}}_{\mathrm{ST}}$ & $\mathrm{n}$ & $\mathrm{F}^{2}$ & GL \\
\hline Acp-1 & 0,132 & 218 & $57,76 * *$ & 26 & 0,014 & 236 & $6,45^{*}$ & 1 \\
\hline Dia-1 & 0,212 & 211 & $178,88^{* *}$ & 50 & 0,123 & 237 & $116,21 * *$ & 2 \\
\hline Est-3 & 0,203 & 208 & $84,41 * *$ & 26 & 0,003 & 230 & 1,40 & 1 \\
\hline Got-1 & 0,011 & 233 & 5,28 & 26 & 0,002 & 264 & 1,06 & 1 \\
\hline G6pdh-1 & 0,029 & 213 & 12,31 & 25 & 0,010 & 244 & $4,93^{*}$ & 1 \\
\hline Lap-1 & 0,158 & 117 & $36,96^{*}$ & 20 & 0,001 & 140 & 0,32 & 1 \\
\hline Mdh-1 & 0,326 & 233 & $151,88^{* *}$ & 26 & 0,287 & 264 & $151,50 * *$ & 1 \\
\hline Mdh-2 & 0,011 & 233 & 5,23 & 26 & 0,002 & 264 & 1,06 & 1 \\
\hline Mdh-3 & 0,161 & 227 & $146,07 * *$ & 52 & 0,002 & 256 & 2,29 & 2 \\
\hline Pgi-1 & 0,011 & 232 & 5,24 & 26 & 0,002 & 263 & 1,05 & 1 \\
\hline Prx-1 & 0,156 & 131 & $81,65 * *$ & 52 & 0,025 & 148 & $15,02 * *$ & 2 \\
\hline Prx-3 & 0,180 & 191 & $137,89 * *$ & 50 & 0,017 & 220 & $15,15^{* *}$ & 2 \\
\hline Skdh-1 & 0,168 & 169 & $56,81 * *$ & 21 & 0,047 & 196 & $18,40^{* *}$ & 1 \\
\hline 6pgdh-1 & 0,015 & 233 & 13,80 & 52 & 0,010 & 263 & $9,94 * *$ & 2 \\
\hline 6pgdh-2 & 0,224 & 230 & $206,43 * *$ & 52 & 0,005 & 260 & 5,17 & 2 \\
\hline
\end{tabular}

cruzamento em C. echinata. A estimativa obtida no arboreto indica que ela é alta $(0,969)$, embora o erro padrão da média sugira que uma pequena taxa de autofecundação $(0,031)$ possa ocorrer e, portanto, que a espécie não é auto-incompatível. A taxa de cruzamento obtida é comparável às estimadas em outras espécies arbóreas tropicais brasileiras climácicas como Esenbeckia leiocarpa Engl. (variação de $\hat{\mathrm{t}}_{\mathrm{m}}=0,925 \mathrm{a}$ 0,997, Seoane et al. 2001) e Eschweilera ovata (Cambess.) Miers (variação de $\hat{\mathrm{t}}_{\mathrm{m}}=0,985$ a 0,999, E. Gusson et al., dados não publicados), bem como a outras espécies arbóreas tropicais como Bertholletia excelsa Humb. \& Bonpl. ( $\left.\hat{\mathrm{t}}_{\mathrm{m}}=0,849\right)$ (O'Malley et al. 1988), Quararibea asterolepis Pitt. $\left(\hat{\mathrm{t}}_{\mathrm{m}}=1,008\right)$ (Murawski et al. 1990), Shorea congestiflora (Thw.) P.S. Ashton $\left(\hat{\mathrm{t}}_{\mathrm{m}}=0,874\right)$ (Murawski et al. 1994), Tachigali versicolor (Standl.) L.O. Williams ( $\hat{\mathrm{t}}_{\mathrm{m}}=0$,998) (Loveless et al. 1998), Cariniana legalis (Mart.) Ktze. (variação de $\hat{\mathrm{t}}_{\mathrm{m}}=0,901$ a 0,990) (Sebbenn et al. 2000) e Chorisia speciosa A. St.-Hil. $\left(\hat{\mathrm{t}}_{\mathrm{m}}=0,816\right)$ (Souza et al. 2003).

A alta taxa de cruzamento observada em C. echinata no arboreto não é surpresa, tendo em vista a alta densidade de plantas compondo o arboreto (262/0,3 ha). Murawski \& Hamrick (1991) comparando a taxa de cruzamento de nove espécies arbóreas tropicais de ocorrência natural com diferentes densidades populacionais observaram a tendência de espécies de baixa densidade apresentarem menor taxa de cruzamento multiloco em comparação a espécies de alta densidade. Espécies que ocorrem em alta densidade populacional aparentemente apresentam maior taxa de cruzamento devido à proximidade das árvores o que pode favorecer o movimento dos polinizadores, em casos de espécies polinizadas por animais, como $C$. echinata. Assim, a alta densidade de plantas no arboreto pode ter influenciado a alta taxa de cruzamento observada. A avaliação do sistema de reprodução em populações naturais de pau-brasil pode melhor esclarecer esta hipótese.

A variação da taxa de cruzamento multiloco individual (0,77 a 1,0) (figura 1), com maior freqüência de valores altos, pode ser o reflexo da heterogeneidade nas frequiências alélicas do pólen cruzado, diferenças na forma de reprodução entre árvores vizinhas, subestrutura genética espacial e diferenças na autoincompatibilidade entre plantas. O comportamento forrageiro dos animais polinizadores, o assincronismo no florescimento, as diferenças na densidade individual de florescimento e a posição espacial das flores podem também causar variações na taxa de cruzamento entre 
Tabela 3. Estimativas de parâmetros do sistema de reprodução em Caesalpinia echinata Lam. implantada em arboreto experimental. [] Intervalo de confiança a 95\% de probabilidade, obtido por 10.000 reamostragens "bootstraps". ( ) Erro padrão da média, obtido por 500 reamostragens "bootstraps".

Table 3. Estimations of the mating system parameters in Caesalpinia echinata Lam. implanted in experimental arboretum. [ ] Level of confidence at $95 \%$ of probability, obtained by 10.000 bootstraps. () Standard deviation obtained by 500 bootstraps.

\begin{tabular}{lc}
\hline Parâmetros & Estimativas \\
\hline Taxa de cruzamento multiloco $\left(\hat{\mathrm{t}}_{\mathrm{m}}\right)$ & $0,969(0,016)$ \\
Taxa de cruzamento uniloco $\left(\hat{\mathrm{t}}_{\mathrm{s}}\right)$ & $0,891(0,019)$ \\
Taxa de cruzamento entre & $0,078(0,015)$ \\
$\quad$ parentes $\left(\hat{\mathrm{t}}_{\mathrm{m}}-\hat{\mathrm{t}}_{\mathrm{s}}\right)$ & $0,081(0,012)$ \\
Correlação de autofecundação $\left(\hat{\mathrm{r}}_{\mathrm{s}}\right)$ & $0,822(0,048)$ \\
Correlação de paternidade $\left(\hat{\mathrm{r}}_{\mathrm{p}}\right)$ & 1,2 \\
№ médio de árvores doadoras de & \\
$\quad$ pólen $\left(1 / \hat{\mathrm{r}}_{\mathrm{p}}\right)$ & $0,169[-0,064 \mathrm{a} 0,375]$ \\
Índice de fixação nas árvores & \\
$\quad$ adultas $\left(\hat{\mathrm{F}}_{\mathrm{f}}\right)$ & $0,095[-0,146 \mathrm{a} 0,324]$ \\
Índice de fixação nas progênies $\left(\hat{\mathrm{F}}_{\mathrm{p}}\right)$ & 0,269 \\
Coancestria dentro de progênies $\left(\hat{\theta}_{\mathrm{xy}}\right)$ & 1,86 \\
Tamanho efetivo de variância $\left(\hat{\mathrm{N}}_{\mathrm{e}(\mathrm{v})}\right)$ & \\
\hline
\end{tabular}

árvores (Murawski 1995).

Taxa de cruzamento entre parentes - A diferença entre a taxa de cruzamento multiloco e a uniloco pode ser usada para inferir sobre a taxa de cruzamento entre indivíduos parentes, visto que a taxa de cruzamento uniloco é esperada ser menor do que a multiloco, diante de progênies advindas de endogamia biparental. Esperase que a taxa de cruzamento uniloco seja viciada para baixo diante de qualquer endogamia adicional à autofecundação, gerando diferença positiva em relação à taxa de cruzamento multiloco, que por sua natureza multiloco é menos susceptível a violações das pressuposições do modelo (Ritland \& Jain 1981, Ritland \& El-Kassaby 1985). A diferença entre as taxas de cruzamentos multiloco e uniloco indicou a presença de $7,8 \%$ de cruzamento entre parentes. Considerando a ausência de informações sobre o número de árvores matrizes que deu origem às sementes utilizadas para a formação do arboreto e o fato de que no Brasil, até hoje, muitos programas de reflorestamentos desconsideram as recomendações sobre o número adequado de árvores para a coleta de sementes, acredita-se que estas tenham sido coletadas de uma ou poucas árvores. Esta hipótese é reforçada pela estimativa do alto índice de fixação observado nas árvores adultas do arboreto (tabela 3). A endogamia em uma geração corresponde a coancestria da geração parental (Lindgren et al. 1996), de forma que o índice de fixação nas árvores adultas corresponde a coancestria entre os parentais que deram origem às sementes que formaram o arboreto, e o índice de fixação medido nas progênies do arboreto corresponde ao coeficiente de coancestria entre as árvores adultas que se cruzaram no arboreto. Assim, o índice de fixação de 0,169 detectado no arboreto sugere que a coancestria entre as árvores que se cruzaram na população de $C$. echinata da Estação Ecológica de Tapacurá encontra-se entre a esperada entre indivíduos meios-irmãos $(0,125)$ e irmãoscompletos $(0,25)$ e o índice de fixação de 0,095 , estimado nas progênies amostradas no arboreto, encontra-se próximo ao esperado entre indivíduos meios-irmãos, ou seja, os resultados indicam que as sementes que deram origem ao arboreto foram geradas em parte por cruzamentos entre indivíduos parentes entre os graus de meios-irmãos e irmãos-completos e foram coletadas de uma ou poucas árvores, caso contrário, o índice de fixação nas progênies teria sido menor. Dessa forma, se as sementes usadas na formação do arboreto foram coletadas de poucas árvores, esperava-se uma taxa maior de cruzamento entre indivíduos parentes no arboreto, comparativamente aos $7,8 \%$ observados.

A ausência de seleção entre o evento de fertilização e o momento de acesso aos genótipos por marcadores genéticos é uma das pressuposições básicas do modelo de reprodução mista de Ritland \& Jain (1981) para a caracterização do sistema de reprodução de uma espécie. Contudo, esta

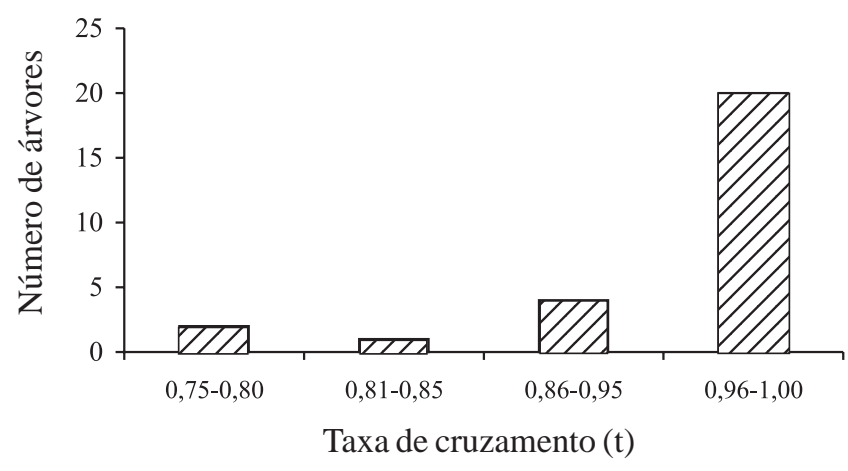

Figura 1. Estimativa da taxa de cruzamento multiloco individual por planta materna $(\hat{\mathrm{t}})$ em Caesalpinia echinata Lam. implantada em arboreto experimental

Figure 1. Estimation of the individual multilocus outcrossing rate $(\hat{t})$ in Caesalpinia echinata Lam. implanted in experimental arboretum. 
pressuposição dificilmente é verdadeira em espécies arbóreas predominantemente de cruzamento, onde os efeitos da depressão endogâmica são fortes (Sørensen 1997). Como conseqüência, em espécies arbóreas, estimativas da taxa de cruzamento, provavelmente, sempre são superestimadas devido à eliminação de parte dos genótipos advindos de autofecundação e de cruzamento entre indivíduos parentes. Por exemplo, Moran \& Brown (1980) observaram em Eucalyptus delegatensis R.T. Bak. que a estimativa da taxa de cruzamento aumentava com o aumento da idade dos frutos retidos nas árvores. A explicação para o fenômeno foi de que as sementes nos frutos estavam sob efeito constante da seleção natural contra endogâmicas. Assim, em frutos mais velhos restariam, praticamente, somente sementes advindas de processos de reprodução que não envolviam endogamia e, por isso, a estimativa da taxa de cruzamento em sementes de tais frutos seria sempre superestimada, comparativamente a sementes coletadas em frutos mais novos. Assim, é possível que a taxa de cruzamento entre parentes calculada em $C$. echinata esteja subestimada e a taxa de cruzamento multiloco esteja superestimada, devido, primeiro, à hipótese de que o arboreto foi formado com sementes coletadas de uma ou poucas árvores matrizes, de forma que deva existir alto grau de parentesco no arboreto e, segundo, devido à alta taxa de mortalidade detectada nas plântulas em relação às sementes coletadas nas árvores matrizes do arboreto [média $=43 \%$, variação de $8,16 \%$ (matriz 11) a 73\% (matriz 25)]. A mortalidade observada em C. echinata pode, em parte, ser atribuída à depressão endogâmica, causada pela endogamia advinda de autofecundações e, especialmente, devido aos cruzamentos entre parentes. Assim, como somente plântulas sobreviventes foram analisadas por eletroforese de isoenzimas e essas são, provavelmente, menos endogâmicas do que todas as sementes produzidas no evento reprodutivo de origem, a taxa de cruzamento pode estar superestimada e a taxa de cruzamento entre parentes pode estar subestimada. Cruzamentos correlacionados - A estimativa da correlação de autofecundação $\left(\hat{r}_{s}\right)$ foi de 0,081 , valor considerado baixo e significativamente diferente de zero (erro padrão da média igual a 0,012) (tabela 3). Esse resultado indica que a variação na taxa de cruzamento entre árvores foi baixa e existe uma certa homogeneidade na estimativa da taxa de cruzamento individual (ver figura 1). Por outro lado, a estimativa da correlação de paternidade $\left(\hat{r}_{p}\right)$ foi 0,822 , valor consideravelmente alto. $\mathrm{O}$ erro padrão da correlação $\hat{r}_{\mathrm{p}}$ demonstra que esta é significativamente diferente de zero e indica que grande parte das progênies amostradas é parente no grau de irmãos-completos. Portanto, indica que o processo de polinização cruzada não foi aleatório e muitos dos cruzamentos eram biparentais (mesmas árvores materna e paterna envolvidas em sucessivos cruzamentos). O número médio de árvores que contribuíram para a polinização foi estimado em apenas 1,2 (tabela 3).

Durante muito tempo acreditou-se que os cruzamentos em populações naturais eram aleatórios. Hoje, ainda, muitos estudos realizados com progênies de polinização aberta, em programas de melhoramento florestal, assumem que essas foram geradas pela contribuição de grande número de árvores polinizadoras e que o parentesco entre plantas dentro de progênies é de meios-irmãos. Os resultados obtidos para a C. echinata no arboreto discordam dessa hipótese. A estimativa da correlação de paternidade indicou que grande parte das progênies advindas de cruzamento foi gerada por cruzamentos biparentais e, portanto, são em sua maioria aparentadas no grau de irmãos-completos. Multiplicando-se a taxa de cruzamento multiloco pela correlação de paternidade determina-se que aproximadamente $80 \%\left(\hat{\mathrm{t}}_{\mathrm{m}} \hat{\mathrm{r}}_{\mathrm{p}}\right)$ das plantas dentro de progênies eram parentes no grau de irmãos-completos e $17 \%$ [ $\left.\hat{\mathrm{t}}_{\mathrm{m}}\left(1-\hat{\mathrm{r}}_{\mathrm{p}}\right)\right]$ eram meios-irmãos. Os cruzamentos correlacionados são causados pelo comportamento dos polinizadores visitando de forma sistemática árvores próximas. Isto explicaria os desvios de cruzamentos aleatórios observados nas freqüências alélicas entre o pólen que fecundou cada árvore.

Em concordância com a alta correlação de paternidade detectada em $C$. echinata, estimativas realizadas com progênies de polinização aberta em outras espécies arbóreas tropicais vêm mostrando que os cruzamentos biparentais são, aparentemente, um fenômeno comum nessas espécies. Em população natural de Cryptocarya moschata Nees (Moraes \& Monteiro 1997), foi detectada correlação de paternidade de 0,990. Em populações de Dryobalanops aromatica Gaertn. f. (Lee 2000), a correlação de paternidade variou de 0,107 a 0,428. Em Enterolobium cyclocarpum Jacq. (Rocha \& Aguilar 2001), a correlação $\hat{r}_{p}$ foi estimada em 0,104 em árvores isoladas em pastagem e de 0,188 em árvores localizadas em floresta. Em C. legalis (Sebbenn et al. 2000), a estimativa da correlação $\hat{\mathrm{r}}_{\mathrm{p}}$ variou de 0,212 a 0,324 e, em Esenbeckia leiocarpa Engl. (Seoane et al. 2001), variou de 0,749 a 0,986. Em Tabebuia cassinoides (Lam.) A.P. DC. (Sebbenn et al. 2001), foi detectada correlação de paternidade de 0,547 
em uma população natural e 0,295 em uma manejada. Em Caryocar brasiliensis Camb. (Collevatti et al. 2001), a estimativa da correlação de paternidade variou de 0,212 a 0,324. Em população natural de Chorisia speciosa (Souza et al. 2003) foi detectada correlação $\widehat{\mathrm{r}}_{\mathrm{p}}$ de 0,875 . Assim, a pressuposição de que espécies arbóreas reproduzem-se por cruzamentos aleatórios, aparentemente, nem sempre é verdadeira, e sua adoção pode incorporar grandes erros nas estimativas de parâmetros genéticos, no estudo da herança de caracteres quantitativos em programas de melhoramento e na determinação de tamanhos amostrais para fins de melhoramento e conservação genética e coleta de sementes para reflorestamentos ambientais.

Da correlação de paternidade é possível determinar o número provável de indivíduos polinizadores, se algumas suposições forem feitas (Ritland 1989). Considerando que existem " $n$ " indivíduos reprodutivos em uma área e que cada um tem a mesma probabilidade de ser o parental masculino (doador de pólen) no cruzamento com uma árvore materna, e se os consecutivos cruzamentos forem independentes (envolvem visitas separadas dos polinizadores), então a probabilidade de que um indivíduo polinizador cruze duas vezes é $1 / \mathrm{n}$. Assim, o número efetivo de polinizadores é $1 / \hat{\mathrm{r}}_{\mathrm{p}}$. Observando a tabela 3 , verifica-se que o número efetivo médio de polinizadores das árvores de C. echinata foi um $(1,2)$. Isto indica que apesar da alta densidade de árvores no arboreto (262 árvores), a dispersão de pólen é restrita na população. Em consequiência, o tamanho efetivo retido em amostras de sementes deve ser baixo, comparado a uma população perfeitamente panmítica.

Índices de fixação, coeficiente de coancestria e tamanho efetivo de variância - $\mathrm{O}$ índice de fixação médio uniloco foi de 0,169 para as árvores adultas $(\hat{\mathrm{F}})$ e 0,095 para as progênies $\left(\hat{\mathrm{F}}_{\mathrm{p}}\right)$. Contudo, de acordo com o intervalo de confiança a $95 \%$ de probabilidade, ambos os valores não são significativamente diferentes de zero. Embora o índice de fixação nas árvores adultas não seja significativo, sua magnitude, 0,169 , tem importante aspecto genético. O índice de fixação positivo, interpretado em termos de coeficiente de endogamia, depende do sistema de reprodução, da endogamia e do parentesco na geração parental. Assim, a endogamia detectada nas árvores adultas sugere que a coancestria entre as árvores parentais na população de origem (Estação Ecológica de Tapacurá, PE) estava entre os graus de meios-irmãos e irmãos-completos. Por outro lado, a menor endogamia nas progênies originadas das árvores do arboreto pode ser explicada, do ponto de vista genético, pela depressão por endogamia. Hipotetiza-se que a seleção natural tenha eliminado grande parte das progênies oriundas de cruzamentos entre parentes e de autofecundações, de forma que parte da endogamia foi eliminada entre a fase de fertilização e a de genotipagem do material.

O coeficiente de coancestria $\left(\hat{\theta}_{x y}\right)$ estimado nas progênies foi de 0,269 , valor consideravelmente alto e superior ao esperado em progênies de meios-irmãos $(0,125)$. Este coeficiente é uma medida de parentesco que mede a probabilidade de amostrar-se aleatoriamente dois alelos em dois indivíduos e eles serem idênticos por descendência. $\mathrm{O}$ significado dessa estimativa para a população em estudo é de que existem $27 \%$ de probabilidade, aproximadamente, de amostrar-se dois alelos em dois indivíduos de mesma progênie e eles serem idênticos por descendência. Essa alta coancestria nas progênies de $C$. echinata é conseqüência do sistema de reprodução, mais especificamente dos cruzamentos biparentais. A endogamia nas árvores adultas também contribuiu para essa coancestria, mas em menor escala do que os cruzamentos biparentais.

O coeficiente de coancestria tem importante papel na estimativa do coeficiente de correlação de parentesco $\left[\begin{array}{ll}r_{x y} & 2 \theta_{x y} /(1 \square f)\end{array}\right]$ entre plantas dentro de progênies, em programas de melhoramento. Em caso de utilização de progênies de polinização aberta de $C$. echinata coletadas do presente arboreto, para fins de melhoramento genético, será necessária a utilização do coeficiente de correlação de 0,538 em substituição ao valor comumente usado de 0,25 , caso contrário, as estimativas de parâmetros genéticos serão altamente superestimadas.

O conhecimento do coeficiente de coancestria também é importante na estimativa do tamanho efetivo de variância $\left(\mathrm{N}_{\mathrm{e}(\mathrm{v})}\right)$, o qual mede a representatividade genética de progênies em relação à população de referência ou parental. Em amostras de estruturas de progênies de tamanho infinito, o tamanho efetivo de variância assume valores entre um (1) e quatro (4). Valor um para progênies de autofecundação, dois para progênies de irmãos-completos e quatro para progênies de meios-irmãos. Assim, em progênies de populações perfeitamente panmíticas, o tamanho efetivo de variância atinge seu valor máximo, quatro (4). A estimativa do tamanho efetivo de variância $\left(\hat{\mathrm{N}}_{\mathrm{e}(\mathrm{v})}\right)$ de uma simples progênie foi baixa, 1,86 (tabela 3), indicando que a representatividade genética na descendência é muito inferior à esperada em progênies de cruzamentos aleatórios. O baixo valor obtido está de acordo com as observações anteriores de que as progênies são 
compostas principalmente por irmãos-completos.

O tamanho efetivo de variância é uma medida de grande valor para a determinação de tamanhos amostrais em programas de melhoramento, conservação genética e para a coleta de sementes visando a recuperação ambiental, bem como para o monitoramento da variabilidade genética em populações sob manipulação. Por exemplo, é possível estimar o número de árvores matrizes $(\mathrm{m})$ necessárias para coleta de sementes do presente arboreto visando reter um determinado tamanho efetivo. Se o objetivo fosse coletar sementes para recuperação ambiental e de cada árvore fossem amostradas 1.000 sementes, para reter um tamanho efetivo de referência mínimo de 50, por exemplo, seria necessário coletar sementes em 27 árvores $\left(\hat{\mathrm{m}}=\hat{\mathrm{N}}_{\mathrm{e}(\text { Referênncia })} / \hat{\mathrm{N}}_{\mathrm{e}(\mathrm{v})}\right)$.

Em suma, os resultados do estudo do sistema de reprodução em C. echinata mostraram que existem altos níveis de endogamia nas árvores adultas do arboreto e que a espécie se reproduz predominantemente por alogamia e, aparentemente, não é auto-incompatível. Também foi observado que os cruzamentos não ocorreram de forma aleatória, sendo parte atribuída a cruzamentos endogâmicos e parte a cruzamentos biparentais. Ainda, observou-se que grande parte das progênies é aparentada no grau de irmãos-completos e que sementes coletadas do arboreto podem ser utilizadas em reflorestamentos, desde que sejam coletadas de um número grande de árvores e após, seja feita uma seleção contra indivíduos que apresentem quaisquer indícios de endogamia.

Agradecimentos - À Fapesp pelo financiamento do projeto (Processo n. 2000/06422-4).

\section{Referências bibliográficas}

AGUIAR, F.F.A. 1992. Comportamento ecológico de Caesalpinia echinata Lam. (Pau-brasil), cultivado em arboreto experimental. Revista Árvore 16:255-261.

ALFENAS, A.C. 1998. Eletroforese de isoenzimas e proteínas afins: fundamentos e aplicações em plantas e microrganismos. Universidade Federal de Viçosa, Viçosa.

ALLARD, R.W. 1971. Princípios do melhoramento genético das plantas. Editora Edgard Blücher, São Paulo.

BRASIL. 1992. Presidência da República. Secretaria do Meio Ambiente - Instituto Brasileiro do Meio Ambiente e dos Recursos Naturais Renováveis. Portaria n $\mathrm{n}^{0}$ 06-N de 15 de Janeiro de 1992. Diário Oficial da União, 23.jan.1992. Seção I, v.130, no 16. Lista Oficial de Espécies da Flora Brasileira Ameaçadas de Extinção.
BURGESS, I.P., WILLIAMS, E.R., BELL, J.C., HARWOOD, C.E. \& OWEN, J.V. 1996. The effect of outcrossing rate on the growth of selected families of Eucalyptus grandis. Silvae Genetica 45:97-100.

CARVALHO, P.E.R. 1994. Espécies florestais brasileiras: recomendações silviculturais, potencialidades e uso de madeira. Embrapa-CNPF, Brasília.

COCKERHAM, C.C. 1969. Variance of gene frequencies. Evolution 23:72-84.

COLLEVATTI, R.G., GRATTAPAGLIA, D. \& HAY, J.D. 2001. High resolution microssatellite based analysis of the mating system allows the detection of significant biparental inbreeding in Caryocar brasiliensis, an endangered tropical tree species. Heredity 86:60-67.

GIUDICE NETO, J. DEL, SEBBENN, A.M. \& KAGEYAMA, P.Y. 2004. Herança e ligação em locos isoenzimáticos de Caesalpinia echinata L. (Pau-brasil). Revista do Instituto Florestal 16:101-110.

KAGEYAMA, P.Y. 1987. Conservação "in situ" de recursos genéticos de plantas. IPEF 35:7-37.

KEPHART, S.R. 1990. Starch gel electrophoresis of plant isozymes: a comparative analysis of techniques. American Journal of Botany 77:693-712.

LEE, S.L. 2000. Mating system parameters of Dryobalanops aromatica Gaertn. f. (Dipterocarpaceae) in three different forest types and a seed orchard. Heredity 85:338-345.

LEWIS, P.O., ZAYKIN, D. 2002. GDA- Genetic Data Analysis: version 1.1 for Windows 95/NT. http:// www.lewis.eeb.uconn.edu/lewishome/software.html (acesso em 19/01/2004).

LINDGREN, D., GEA, L. \& JEFFERSON, P. 1996. Loss of genetic diversity by status number. Silvae Genetica 45:52-59.

LOVELESS, M.D., HAMRICK, J.L. \& FOSTER, R.B. 1998. Population structure and mating system in Tachigali versicolor a monocarpic neotropical tree. Heredity 35:134-143.

MORAES, P.L.R. \& MONTEIRO, R. 1997. Taxas de cruzamento em uma população natural de Cryptocarya moschata Nees. (Lauraceae). Biota Neotropica v2 (n2) http://www.biotaneotropica.org.br/v2n $2 / \mathrm{pt} /$ abstract?article+BN01102022002 (Acesso em 05/04/ 2004).

MORAN, G.F. \& BROWN, A.H.D. 1980. Temporal heterogenity of outcrossing rates in Alpine Ash (Eucalyptus delegatensis R.T. Bak.). Theoretical and Applied Genetics 57:101-105.

MURAWSKI, D.A. 1995. Reproductive biology and genetics of tropical trees from canopy perspective. In: Forest canopies (M.D.Lowman \& N.M.Nadkarni, eds.). Academic Press, New York, p.457-493.

MURAWSKI, D.A. \& HAMRICK, J.L. 1991. The effect of the density of flowering individuals on the mating systems of nine tropical tree species. Heredity 67:167-174. 
MURAWSKI, D.A., HAMRICK, J.L., HUBBELL, S.P. \& FOSTER, R.B. 1990. Mating system of two Bombacaceous trees of a neotropical moist forest. Oecologia 82:501-506.

MURAWSKI, D.A., DAYANANDAN, B. \& BAWA, K.S. 1994. Outcrossing rates of two endemic Shorea species from Sri Lankan Tropical Rain Forests. Biotropica 26:23-29.

NEI, M. 1977. F-statistics and analysis of gene diversity in subdivided populations. Annals of Human Genetics 41:225-233.

O'MALLEY, D.M., BUCKLEY, D.P., PRANCE, G.T. \& BAWA, K.S. 1988. Genetic of Brazil nut (Bertholletia excelsa Humb. \& Bonpl.: Lecythidaceae). 2. Mating system. Theoretical and Applied Genetics 76: 929-932.

RITLAND, K. 1989. Correlated mating in the partial selfer Mimulus guttatus. Evolution 43:848-859.

RITLAND, K. 1994. Multilocus mating system program MLTR. Version 1.1. Departament of Forest Science, University of British Columbia, Vancouver.

RITLAND, K. \& EL-KASSABY, Y.A. 1985. The nature of inbreeding in a seed orchard of Douglas fir as shown by an efficient multilocus model. Theoretical and Applied Genetics 71:375-384.

RITLAND, K. \& JAIN, S. 1981. A model for the estimation of outcrossing rate and gene frequencies using independent loci. Heredity 47:35-52.

ROCHA, O.J.R. \& AGUILAR, G. 2001. Variation in the breeding behavior of the dry forest tree Enterolobium cyclocarpum (Guanacaste) in Costa Rica. American Journal of Botany 89:1600-1606.
SEBBENN, A.M. 2002. Número de árvores matrizes e conceitos genéticos na coleta de sementes para reflorestamentos com espécies nativas. Revista do Instituto Florestal 14:115-132.

SEBBENN, A.M., KAGEYAMA, P.Y., SIQUEIRA, A.C.M.F. \& ZANATTO, A.C.E. 2000. Taxa de cruzamento em populações de Cariniana legalis (Mart.) O. Ktze.: Implicações para a conservação e o melhoramento genético. Scientia Forestalis 58:25-40.

SEBBENN, A.M., SEOANE, C.E.S., KAGEYAMA, P.Y. \& LACERDA, C.M.B. 2001. Estrutura genética em populações de Tabebuia cassinoides: Implicações para o manejo florestal e a conservação genética. Revista do Instituto Florestal 13:93-113.

SEOANE, C.E.S., SEBBENN, A.M. \& KAGEYAMA, P.Y 2001. Sistema reprodutivo em populações de Esenbeckia leiocarpa. Revista do Instituto Florestal 13:19-26.

SOLTIS, D.E. \& SOLTIS, P.S. 1989. Isozymes in plant biology. Dioscorides Press, Portland.

SØRENSEN, F.C. 1997. Effects of sib mating and wind pollination on nursery seedling size, growth components, and phenology of Douglas-Fir seedorchard progenies. Canadian Journal of Forest Research 27:557-566.

SOUZA, L.M.I., SEBBENN, A.M. \& KAGEYAMA, P.Y. 2003. Sistema de reprodução em Chorisia speciosa. Revista Brasileira de Botânica 26:113-121.

WORKMAN, P. \& NISWANDER, J.L. 1970. Population studies on southwestern Indian Tribes. II. Local genetic differentiation in the Papago. The American Journal of Human Genetics 22:24-49. 